\title{
The Leloir Pathway of Galactose Metabolism - A Novel Therapeutic Target for Hepatocellular Carcinoma
}

\author{
MANSHU TANG, ENOABASI ETOKIDEM and KENT LAI \\ Division of Medical Genetics, Department of Pediatrics, \\ University of Utah School of Medicine, Salt Lake City, UT, U.S.A.
}

\begin{abstract}
Hepatocellular carcinoma (HCC) is one of the most lethal types of cancer worldwide, with poor prognosis and limited treatments. In order to identify novel therapeutic targets that will lead to development of effective therapies with manageable side effects, we tested the hypothesis that knocking-down galactokinase (GALK1) or galactose-1 phosphate uridylyltransferase (GALT) gene expression would control the growth of cultured hepatoma cells. Our results showed small interfering RNA (siRNA) against GALK1 or GALT inhibited the growth of HepG2 cells in culture. Western blot analysis revealed simultaneous down-regulation of multiple players of the phosphatidylinositol 3-kinase $(P I 3 K) /$ protein kinase $B(A K T)$ growth signaling pathway, as well as heat-shock protein 90 (HSP90) and poly ADP ribose polymerase $(P A R P)$. Reverse transcription-polymerase chain reaction (RT-PCR) data, however, showed no significant mRNA reduction of the encoded genes. Our study thus not only supports GALK1 and GALT as being possible novel targets for treating $\mathrm{HCC}$, but also uncovers new posttranscriptional regulatory mechanisms that link the galactose metabolic pathway to protein expression of the PI3K/AKT pathway in hepatoma.
\end{abstract}

Cancer is one of the leading causes of mortality and morbidity in both developing and developed nations. The American Cancer Society estimates that in 2016, there will be 1,685,210 new cancer cases diagnosed and 595,690 cancer deaths in the U.S. alone (1). Despite significant progress in anticancer therapies, many types of cancer, such as liver cancer, hepatocellular carcinoma (HCC) in particular,

Correspondence to: Manshu Tang or Kent Lai, Division of Medical Genetics, Department of Pediatrics, University of Utah School of Medicine, 295 Chipeta Way, Salt Lake City, UT 84108, U.S.A. Email: manshu.tang@hsc.utah.edu or kent.lai@hsc.utah.edu

Key Words: Galactose metabolism, hepatocellular carcinoma, siRNA, HSP90, PI3K/AKT, PARP, galactokinase, galactose -1 phosphate uridylyltransferase, HepG2. continue to have poor prognosis (2). Moreover, the deleterious side-effects associated with even the most advanced types of cytotoxic chemotherapies often take a toll on the quality of life of the patients. Recent research efforts have switched from broad-based chemotherapy to targeted therapy in an attempt to minimize treatment toxicities (3); and emerging research shows that altered cellular metabolism in cancer cells can serve as new sites for targeted intervention (4-6).

In humans, metabolism of galactose absorbed from digested foodstuffs, as well as that produced endogenously, begins with the phosphorylation of hexose by the enzyme galactokinase (GALK1) to form galactose-1 phosphate (gal1P) (Figure 1). In the presence of another enzyme, galactose1 phosphate uridylyltransferase (GALT), gal-1P will then react with uridine diphosphate glucose (UDP-glucose) to form glucose-1 phosphate (glu-1P) and uridine diphosphate galactose (UDP-galactose) (Figure 1). UDP-galactose is a galactosyl donor for the biosynthesis of glycoproteins and glycolipids, which are crucial for many cancer cellular functions such as cell-cell recognition, metastasis and receptor-mediated signaling (7-11). UDP-galactose can also be formed directly from UDP-glucose via the action of UDPgalactose 4'-epimerase (GALE) (Figure 1), but this reaction alone is unlikely to be efficient enough to meet the cell's demand for UDP-galactose.

When compared to normal liver tissues, there is significant up-regulation of GALK1 and GALT genes in human liver cancer tissues, indicating the higher demand for the products of the GALK1 and GALT enzymes by these cancer cells. Barretina and co-workers reported that the GALK1 gene was up-regulated by at least six-fold in 28 different human liver cancer cell lines, while the GALT gene was up-regulated by eight-fold or more (12). Given the genetic heterogeneity of liver cancer (13), the up-regulation seen in 28 different cell lines examined is highly significant. In addition, our recent work on the mouse model of human GALT deficiency revealed a positive regulatory role of GALT gene function in the expression of the phosphatidylinositol 3-kinase $(\mathrm{PI} 3 \mathrm{~K}) /$ protein kinase $\mathrm{B}$ (AKT) growth signaling pathway 


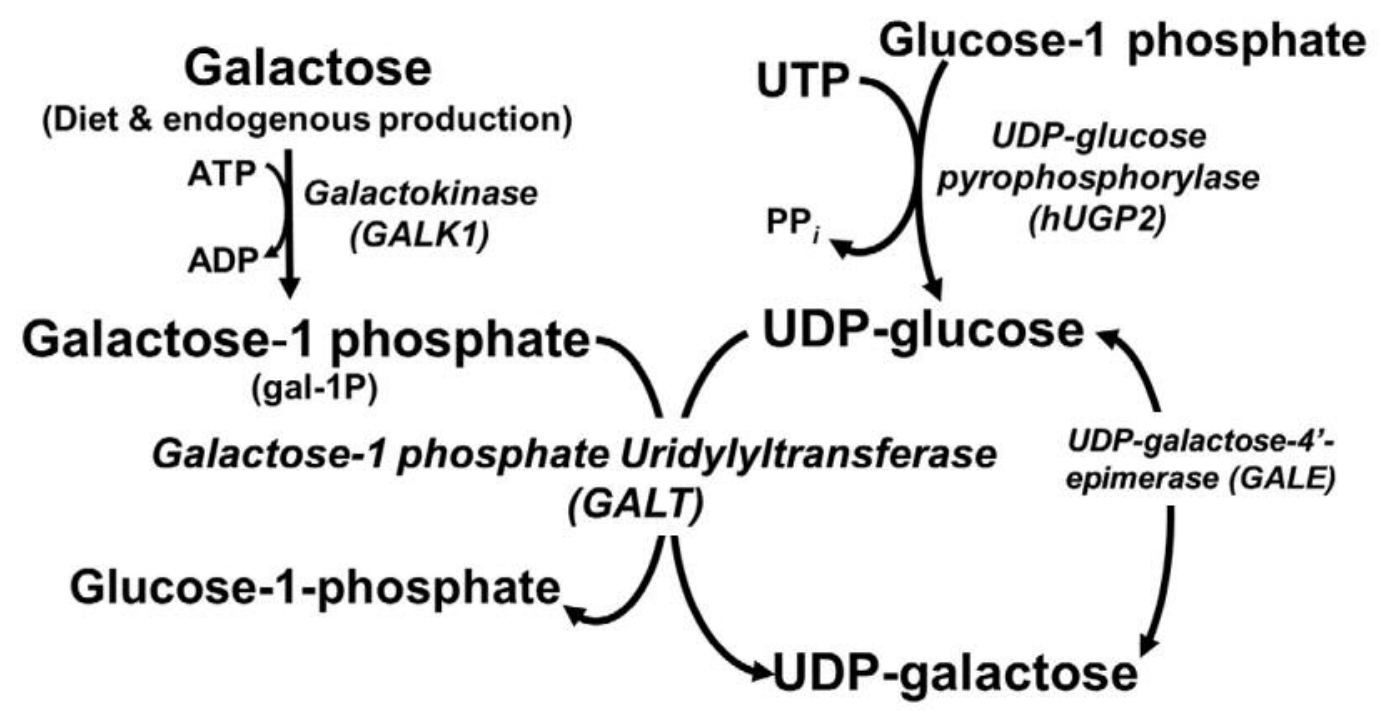

Figure 1. The Leloir pathway of galactose metabolism. Under normal conditions, exogenous and endogenously-produced galactose is phosphorylated by galactokinase (GALK1) to form galactose-1-phosphate (gal-1P). In the presence of galactose-1-phosphate uridylyltransferase (GALT), gal-1P reacts with UDP-glucose to form glucose-1-phosphate and UDP-galactose. UDP-galactose can also be formed from UDP-glucose via the UDP galactose-4'-epimerase (GALE) reaction.

(14), which in turn plays a significant role in the growth and progression of human HCC (15). However, no GALK1deficient mouse models are available for similar studies.

Deficiencies of GALK1 and GALT enzymatic functions have relatively benign and manageable consequences to normal, noncancerous cells, which is crucial for preserving the quality of life for the patients under treatment. For example, patients with inherited deficiency of GALK1 (type II galactosemia) have increased incidence of pre-senile cataracts, but no lifethreatening or other serious consequences (16). Cataract formation often self-resolves when the patient is put on a galactose-restricted diet. Some patients with inherited severe GALT deficiency (type I galactosemia, 0\% GALT activity) might develop long-term complications such as ataxia $(20 \%$ incidence) and primary ovarian insufficiency (92\% incidence) $(17,18)$, but these complications are unlikely to pose serious concerns for older patents with terminal liver cancer.

In this study, we applied small interfering RNA (siRNA) targeting GALK1 and GALT genes in the HCC model cell line HepG2 to test the hypothesis that knocking-down expression of GAL genes would have negative impact on the growth of the cultured hepatoma cells. Successful validation of these targets will pave the way for formal development of therapies.

\section{Materials and Methods}

Materials. HepG2 cell line was obtained from the American Type Culture Collection (Manassas, VA USA) and maintained in Dulbecco's modified Eagle's medium (DMEM) supplemented with
$10 \%$ fetal bovine serum and penicillin/streptomycin. Antibody to glyceraldehyde 3-phosphate dehydrogenase (GAPDH) was purchased from Santa Cruz Biotechnology, Inc. (Santa Cruz, CA, USA). Antibodies against phosphorylated AKT (Ser473) (p-AKT Ser473), phosphorylated AKT(Thr308) (p-AKT Thr308), total AKT (pan AKT), phosphorylated phosphatase and tensin homolog (Ser380) (p-PTEN Ser380), phosphorylated phosphoinositidedependent kinase 1 (Ser241) (p-PDK1 Ser241), phosphorylated glycogen synthase kinase $3 \beta$ (Ser9) (p-GSK3 $\beta$ Ser9), heat-shock protein 90 (HSP90) and poly ADP ribose polymerase (PARP) were purchased from Cell Signaling Technology, Inc. (Danvers, MA, USA). Fluorescently labeled secondary antibodies were purchased from Li-Cor Biosciences (Lincoln, NE, USA). Lipofectamin RNAiMax, RNeasy mini kit, Superscript RT kit, polyT primer, Taqman 2× Master buffer, primers and probes for $A K T, P D K 1$, $G A P D H, P A R P, G A L T$ and $G A L K 1$ were purchased from Invitrogen (Carlsbad, CA, USA). siRNAs were also synthesized by Invitrogen.

Sequences for siRNAs. GALK1: GGGCCUUCUAUGUCAA GGGAGUGAU; GALT: GUCUCCACGCGCAGUACAGUUGC UU; Control: GUCUCCACGCGCAGUACAGUUGCUU.

siRNA transfection and growth monitoring of cultured HepG2 cells. HepG2 cells were plated in replicates in 12-well plates at a density of $5 \times 10^{3}$ cells/well at day 0 . Soon after the cells settled, siRNAs against GALK1, GALT and control siRNA were transfected into the cells in multiple wells with Lipofectamin RNAiMax at $30 \mathrm{nM}$ according to the manufacturer's protocol on the same day. The cells were then trypsinized from the respective wells and counted with a hemocytometer every other day. At day 4, another transfection was carried out in the same way as the first transfection.

Western blot analysis. HepG2 cells were collected at day 8 in lysis buffer [25 mM HEPES (pH 7.5), $120 \mathrm{mM} \mathrm{NaCl,} 1 \mathrm{mM}$ EDTA, 


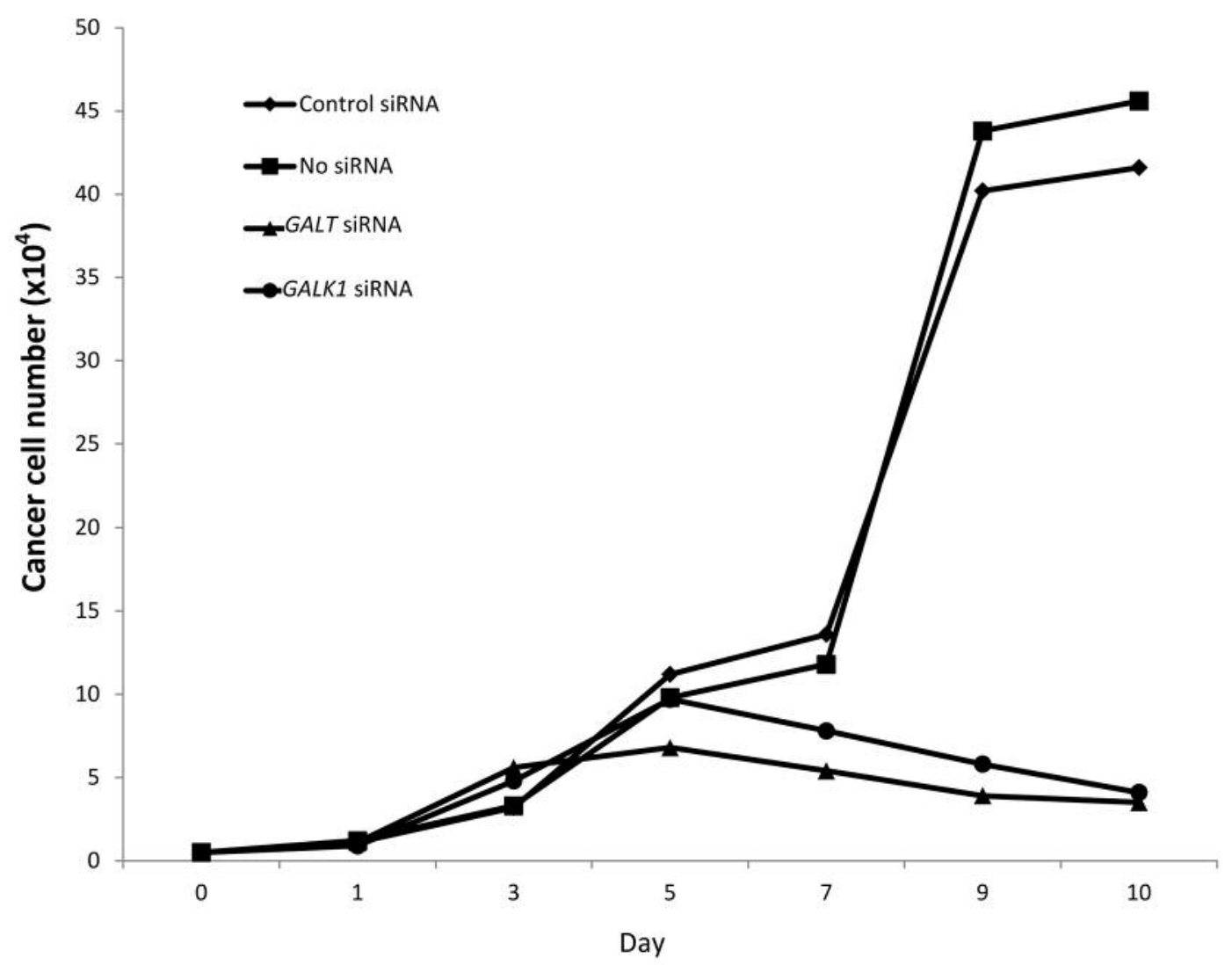

Figure 2. Inhibition of HepG2 cell growth by siRNAs against both galactokinase (GALK1) and galactose-1-phosphate uridylyltransferase (GALT) genes. HepG2 cells were seeded in multiple wells at $5 \times 10^{3}$ per well in a 12-well plate at day 0. After the cells settled, different siRNA treatments were applied. Cell growth was monitored by counting cells every other day.

$10 \mathrm{mM}$ pyrophosphate, $10 \mathrm{mM}$ glycerophosphate, $50 \mathrm{mM} \mathrm{NaF}$, $0.5 \mathrm{mM}$ orthovanadate, and EDTA-free protease inhibitors; Roche Applied Science, Indianapolis, IN, USA]. After centrifugation at $13,000 \times g$ for $10 \mathrm{~min}$, samples containing $20-50 \mu \mathrm{g}$ of protein were resolved by sodium dodecyl sulfate-polyacrylamide gel electrophoresis, and proteins were transferred to nitrocellulose membrane (Bio-Rad Laboratories, Hercules, CA, USA), blocked in $5 \%$ non-fat dried milk, and incubated with the primary antibodies for p-AKT Ser473, p-AKT Thr308, pan AKT, p-PDK1 Ser241, pPTEN Ser308, p- GSK3 $\beta$ Ser9, HSP90, PARP and GAPDH overnight at $4{ }^{\circ} \mathrm{C}$. The blots were washed with wash buffer and secondary antibodies were applied for $1 \mathrm{~h}$ at room temperature. Then the blots were scanned with Odyssey scanner and the analysis was performed on Image Studio Lite (both Li-Cor Biosciences).

Reverse transcription polymerase chain reaction ( $R T-P C R)$. RNA extraction, reverse transcription for the same samples used above were carried out according to the manufacturer's protocol. The cDNA was mixed with $2 \times$ TaqMan Master mix and primer/probe mix for AKT, PDK1, PARP, GALK1, GALT and GAPDH individually in 384-well PCR plate, and the RT-PCR was carried out in a 7900HT RT-PCR system from Applied Biosystems (Carlsbad, CA, USA), the data was imported to QuantStudio 12K Flex Software from Applied Biosystems (Carlsbad, CA, USA) for analysis.

\section{Results}

Knock-down of GALK1 and GALT genes by siRNA inhibited the growth of cultured HepG2 cells. The growth of HepG2 cells treated with either the control or siRNAs against the two GAL genes had no apparent difference at the early stage of the experiment (day 1-5). But at day 6, growth of HepG2 cells treated with siRNAs targeting GALK1 and GALT genes plateaued and the cell numbers started to decrease gradually, while cells of the control groups grew aggressively and reached $40 \times 10^{4}$ cells at the same time frame, as shown in Figure 2. Growth inhibition continued for another 4 days.

siRNAs against GALK1 and GALT genes down-regulated PI3K/AKT signaling pathway. Our previous study of the skin fibroblasts derived from a mouse model of classic galactosemia revealed that GALT deficiency resulted in impaired PI3K/AKT signaling pathway in these cells (14). We investigated whether the same would hold true for cultured HepG2 cells. As shown in Figure 3a, treatment with GALT siRNA also induced the attenuation of PI3K/AKT 
a

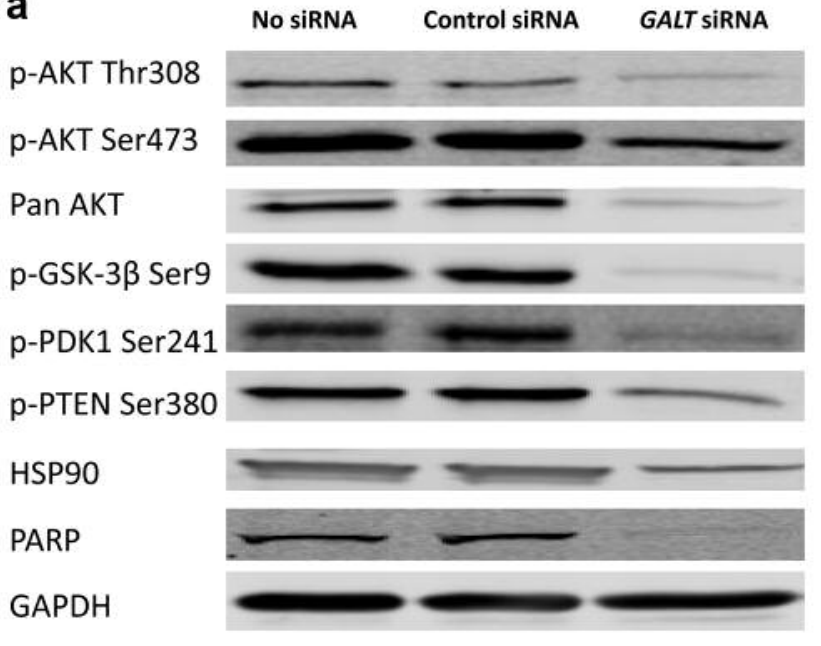

b p-AKT Thr308

p-AKT Ser473

Pan AKT

p-GSK-3 $\beta$ Ser9

p-PDK1 Ser241

p-PTEN Ser380

HSP9O

PARP

GAPDH
No siRNA Control siRNA GALK1 siRNA
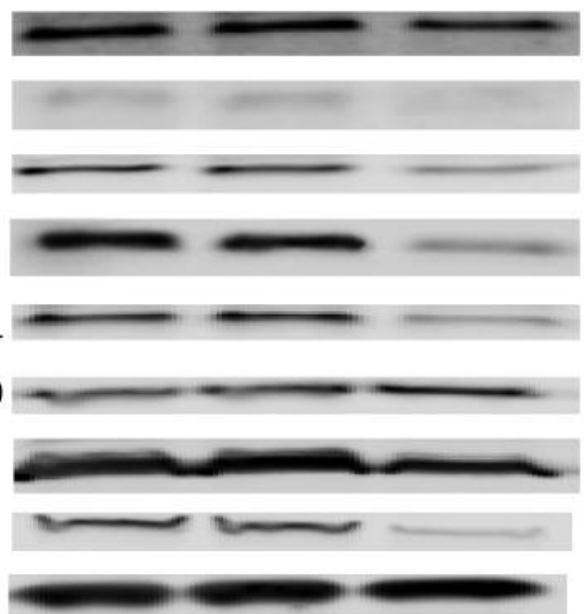

Figure 3. Knock-down of galactokinase (GALK1) and galactose-1-phosphate uridylyltransferase (GALT) gene expression led to down-regulation of the phosphatidylinositol 3-kinase (PI3K)/protein kinase B (AKT) signaling pathway. Western blot analyses of HepG2 cells at day 8 after treatment with GALT siRNA (a) and with GALK1 siRNA (b).

signaling pathway. Abundances for both forms of phosphorylated AKT decreased when the HepG2 cells were treated with GALT siRNA, as well as the total AKT level, which in turn caused the down-regulation of phosphorylated GSK3 $\beta$. Both the positive and negative players of PI3K/AKT signaling pathway, pPDK1 and pPTEN, respectively, were also down-regulated. In addition, the levels of HSP90 and PARP also decreased.

Similarly, when the HepG2 cells were treated with GALK1 siRNA, the levels of all the players of PI3K/AKT signaling pathway, except pPTEN, decreased (Figure 3b). The expression level of HSP90 and PARP were also down-regulated.

siRNAs targeting GALT and GALK1 down-regulated AKT pathway at the post-transcriptional level. In order to confirm the efficiency of siRNAs, we performed RT-PCR assays on the same samples used above. As expected, mRNA levels for both $G A L K 1$ and GALT were down-regulated by over $80 \%$ by their respective siRNAs (Figure 4). Interestingly, the mRNA levels for $A K T, P D K 1$ and $P A R P$ did not change significantly upon treatment with $G A L T$ siRNA nor $G A L K 1$ siRNA, which suggests the attenuation of the AKT pathway by these two siRNAs is through post-transcriptional mechanisms.

\section{Discussion}

HCC is a leading cause of cancer death worldwide (GLOBOCAN 2012, http://globocan.iarc.fr), and is the most rapidly growing cause of cancer-related mortality in the U.S. In contrast to developing countries, in which hepatitis B virus infection is the major cause of $\mathrm{HCC}$, chronic infection with hepatitis $\mathrm{C}$ virus is the leading risk factor for the increasing incidence in developed countries (19). In both scenarios, abnormal activation of the PI3K/AKT pathway has been found in tumor cells $(20,21)$. Studies have also shown that chronic activation of the PI3K/AKT pathway due to loss of PTEN leads to HCC in transgenic mouse models (22), and inhibition of this pathway by sorafenib or rapamycin greatly slowed down HCC progression in animal models (23).

In this study, we demonstrated that disruption of the galactose metabolism pathway by siRNAs against both GALT, and GALK1 inhibited the growth of HepG2 cells in culture (Figure 2). Further analysis revealed that one of the most important cellular growth signal pathways, the PI3K/AKT pathway, was greatly attenuated in siRNA-treated cells. Our findings are significant because of multiple reasons. Firstly, we uncovered the link between the galactose metabolic pathway and the PI3K/AKT signaling pathway in these liverspecific cancerous cells. Secondly, the discordance between the reduced protein abundance of the members of the PI3K/AKT pathway (e.g. AKT, pPDK1 and pGSK-3 $\beta$ ) (Figure 3 ) and the unchanged mRNA levels of their encoding genes (Figure 4) revealed a novel post-transcriptional regulatory mechanism for these protein members.

Thirdly, since the abundance of HSP90 also decreased upon treatment with GALT or GALK1 siRNA (Figure 4), we hypothesize that the post-transcriptional down-regulation of PI3K/AKT pathway is through affecting the abundance of HSP90, as AKT, pPDK1 and pGSK-3 $\beta$ are all clients of 

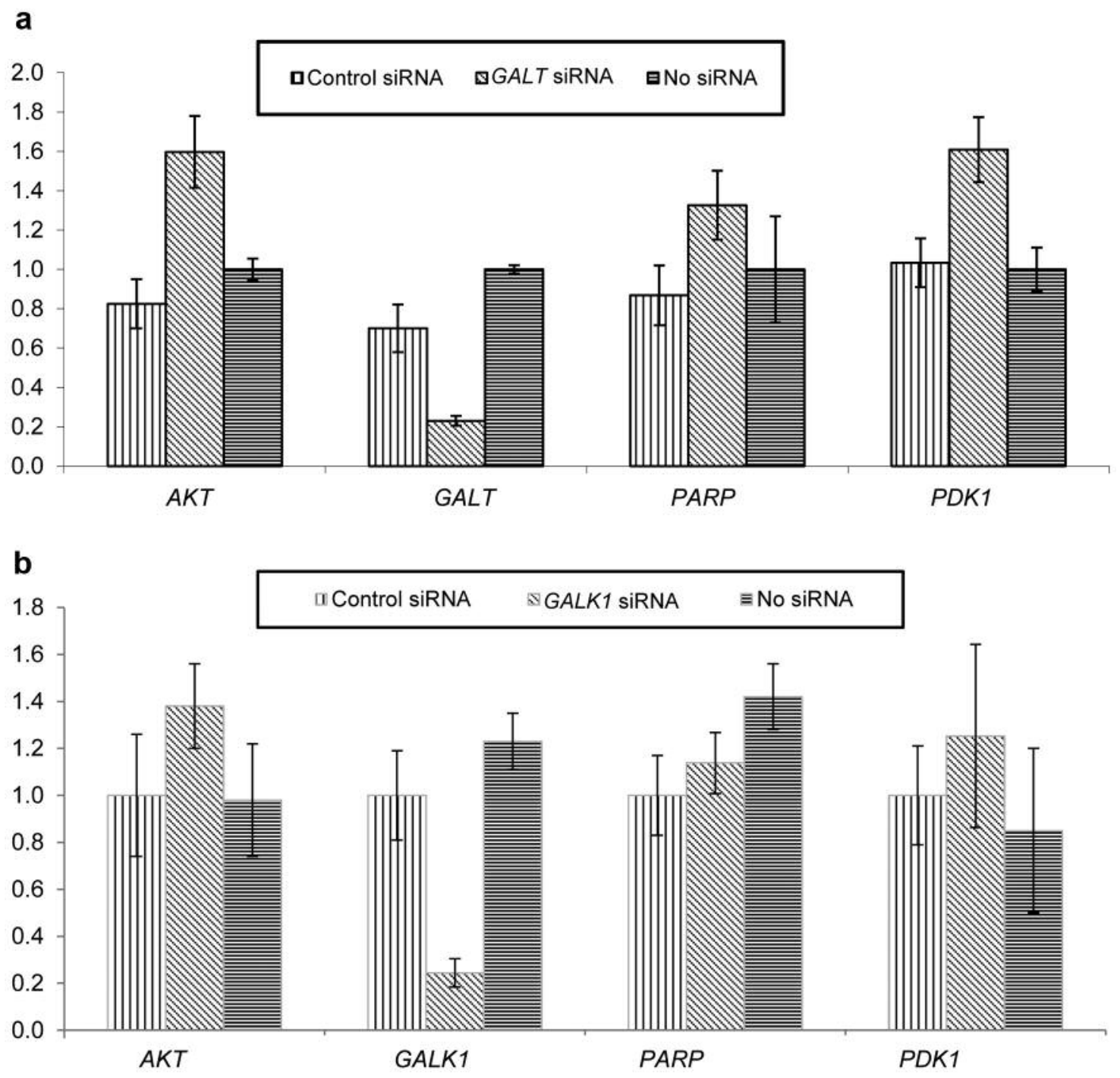

Figure 4. Reverse transcription-polymerase chain reaction (RT-PCR) assays for HepG2 cells treated with galactose-1-phosphate uridylyltransferase $(G A L T)(a)$ and galactokinase $(G A L K 1)(b)$ siRNAs. HepG2 cells were harvested at day 8 after siRNA treatment and mRNA levels of protein kinase $B(A K T)$, phosphoinositide-dependent kinase 1 (PDK1), poly ADP ribose polymerase (PARP), GALK1 and GALT were detected through RT-PCR.

HSP90, and the correct folding and maturation of these proteins are dependent on the proper functioning of HSP90. HSP90 is the most abundant chaperone protein in cells, being constitutively expressed, and can be further induced upon heat shock or other stress stimuli. It accounts for $1-2 \%$ of the total protein in unstressed cells, which increases to $4-6 \%$ under stress (24). As a chaperone, HSP90 assists folding of more than 200 client proteins (25), including many protein kinases, transcription factors, such as AKT, PDK1, mechanistic target of rapamycin (mTOR) and GSK-3 $\beta$ (http://www.picard.ch/downloads/Hsp90interactors.pdf.). HSP90 plays a crucial role in almost all hallmark aspects of oncogenesis, such as self-sufficiency in growth signaling, evasion of death signals and angiogenesis (26-28). Thus inhibition of HSP90 functions has been regarded as a broader anticancer therapy approach rather than antagonists against a single signaling pathway. Not surprisingly, HSP90 inhibitors showed great potential in HCC in many studies (29-31). If our hypothesis is correct, we have found a new regulatory link between the galactose metabolic pathway and abundance of HSP90 in cancer cells. This link can be exploited to complement other existing anti-HSP90 therapies.

Fourthly, we discovered that one of the key DNA damagerepair proteins, PARP, was also down-regulated upon treatment with both GALK1, and GALT siRNA. PARP binds to DNA strand breaks and nicks upon DNA damage and 
mediates the synthesis of PAR polymers from NAD, causing poly(ADP)-ribosylation of itself and other proteins to recruit other factors in DNA-damage repair (32-34). Its role in sensing DNA damage and initiating base excision repair of single-stranded breaks makes it an attractive anticancer target. PARP inhibitors have been investigated as potentiators of chemotherapy and radiation therapy for a large variety of solid malignancies, including breast, pancreatic, glioma, nonsmall cell lung, and melanoma $(35,36)$. PARP is not a client of HSP90, and we know of no reported case that these two proteins were down-regulated simultaneously in any biological event so far. Our results demonstrate, as far as we are aware for the first time, that targeting galactose metabolism pathway through GALKI and GALT siRNAs can simultaneously down-regulate HSP90 and PARP, which play key roles in homeostasis of cancer cells at both protein and DNA levels. It is unlikely that this finding was caused by off-target effect of siRNAs, as two different siRNAs against two different targets generated the same results.

As to the mechanism of how knocking-down GALT and GALK1 have such profound effects on HepG2 cells, we believe that protein glycosylation might play an important role. As cancer cells grow at a much faster pace than normal cells, the demand for protein synthesis and protein glycosylation are much higher than normal cells. Early studies showed that in patients with classic galactosemia who are deficient in GALT, the substrates for protein glycosylation, UDP-glucose and UDP-galactose, are significantly reduced (37). Other studies have shown that interrupting protein glycosylation caused down-regulation of the PI3K/AKT pathway and cell growth arrest $(38,39)$. However, we cannot preclude possibilities of other mechanisms which may lead to the down-regulation of PI3K/AKT, HSP90 and PARP in cancer cells upon knocking-down GALKI and GALT.

Currently, sorafenib is the only selective chemotherapy for $\mathrm{HCC}$ and is one of the standard treatments for advance-stage HCC. Yet sorafenib still has many debilitating side-effects, including fatigue, rash, loss of appetite, diarrhea, high blood pressure, and redness, pain, swelling, or blisters on the palms of the hands or soles of the feet (40). Our observations validate GALK1 and GALT as candidate targets for novel treatment for HCC. We expect that knocking-down these two targets will have minimum, manageable side-effects based on the studies of different types of galactosemia. In classic galactosemia in which patients have complete GALT deficiency, primary ovarian insufficiency and ataxia are major concerns $(17,18)$. But as we have shown in this study, we did not need to eliminate GALT expression entirely with GALT siRNA in order to achieve an effect. In fact, with only about $80 \%$ knock-down, we induced a profound impact on the PI3K/AKT pathway, HSP90 and PARP in HepG2 cells (Figures 3 and 4). Regarding the possibly side-effect of GALK1 deficiency, the only concern is cataracts, which can easily be removed through surgery (16). Based on these observations and facts, we believe that GALK1 and GALT are novel and safer therapeutic targets for HCC.

\section{Acknowledgements}

Grant support to KL included 1R01HD074844 (NIH/NICHD) and the K2R2R award from the Primary Children's Hospital Foundation (Intermountain Healthcare).

\section{References}

$1 \mathrm{http} / / /$ www.cancer.org/research/cancerfactsstatistics/cancer facts figures2016/

2 Motola-Kuba D, Zamora-Valdes D, Uribe $M$ and MendezSanchez N: Hepatocellular carcinoma. An overview. Ann Hepatol 5(1): 16-24, 2006.

3 Gerber DE: Targeted therapies: A new generation of cancer treatments. Am Fam Physician 77(3): 311-319, 2008.

4 Ferreira LM: Cancer metabolism: The Warburg effect today. Exp Mol Pathol 89(3): 372-380, 2010.

5 Hsu PP and Sabatini DM: Cancer cell metabolism: Warburg and beyond. Cell 134(5): 703-707, 2008.

6 Vander Heiden MG: Targeting cancer metabolism: A therapeutic window opens. Nat Rev Drug Discov 10(9): 671-684, 2011.

7 Connelly MA, Gruppen EG, Otvos JD and Dullaart RP: Inflammatory glycoproteins in cardiometabolic disorders, autoimmune diseases and cancer. Clin Chim Acta 459: 177-186, 2016.

8 Nicastri A, Gaspari M, Sacco R, Elia L, Gabriele C, Romano R, Rizzuto A and Cuda G: $N$-Glycoprotein analysis discovers new up-regulated glycoproteins in colorectal cancer tissue. J Proteome Res 13(11): 4932-4941, 2014.

9 Thakkar V, Patel P, Prajapati N, Kaur R and Nandave M: Serum levels of glycoproteins are elevated in patients with ovarian cancer. Indian J Clin Biochem 29(3): 345-350, 2014.

10 Bhavanandan VP: Cancer-associated mucins and mucin-type glycoproteins. Glycobiology 1(5): 493-503, 1991.

11 Muramatsu T: Glycoproteins associated with metastatic potential of cancer. Gan To Kagaku Ryoho 16(10): 3354-3358, 1989 (in Japanese).

12 Barretina J, Caponigro G, Stransky N, Venkatesan K, Margolin AA, Kim S, Wilson CJ, Lehar J, Kryukov GV, Sonkin D, Reddy A, Liu M, Murray L, Berger MF, Monahan JE, Morais P, Meltzer J, Korejwa A, Jane-Valbuena J, Mapa FA, Thibault J, Bric-Furlong E, Raman P, Shipway A, Engels IH, Cheng J, Yu GK, Yu J, Aspesi P, Jr., de Silva M, Jagtap K, Jones MD, Wang L, Hatton C, Palescandolo E, Gupta S, Mahan S, Sougnez C, Onofrio RC, Liefeld T, MacConaill L, Winckler W, Reich M, Li N, Mesirov JP, Gabriel SB, Getz G, Ardlie K, Chan V, Myer VE, Weber BL, Porter J, Warmuth M, Finan P, Harris JL, Meyerson M, Golub TR, Morrissey MP, Sellers WR, Schlegel R and Garraway LA: The cancer cell line encyclopedia enables predictive modelling of anticancer drug sensitivity. Nature 483(7391): 603-607, 2012.

13 Caviglia JM and Schwabe RF: Mouse models of liver cancer. Methods Mol Biol 1267: 165-183, 2015.

14 Balakrishnan B, Chen W, Tang M, Huang X, Cakici DD, Siddiqi A, Berry G and Lai K: Galactose-1 phosphate uridylyltransferase (GALT) gene: A novel positive regulator of the PI3K/AKT 
signaling pathway in mouse fibroblasts. Biochem Biophys Res Commun 470(1): 205-212, 2016.

15 Gedaly R, Angulo P, Hundley J, Daily MF, Chen C and Evers BM: PKI-587 and sorafenib targeting PI3K/AKT/MTOR and RAS/RAF/MAPK pathways synergistically inhibit hoc cell proliferation. J Surg Res 176(2): 542-548, 2012.

16 Bosch AM, Bakker HD, van Gennip AH, van Kempen JV, Wanders RJ and Wijburg FA: Clinical features of galactokinase deficiency: A review of the literature. J Inherit Metab Dis 25(8): 629-634, 2002.

17 Waggoner D, Buist, NRM: Long-term complications in treated galactosemia - 175 U.S. Cases. International Pediatrics 8: 97100, 1993.

18 Waggoner DD, Buist NRM and Donnell GN: Long-term prognosis in galactosemia: Results of a survey of 350 cases. J Inherit Metab Dis 13: 802-818, 1990.

19 El-Serag HB: Hepatocellular carcinoma. N Engl J Med 365(12): 1118-1127, 2011.

20 Nakamura H, Aoki H, Hino O and Moriyama M: HCV core protein promotes heparin binding EGF-like growth factor expression and activates AKT. Hepatol Res 41(5): 455-462, 2011

21 Boyault S, Rickman DS, de Reynies A, Balabaud C, Rebouissou S, Jeannot E, Herault A, Saric J, Belghiti J, Franco D, BioulacSage P, Laurent-Puig P and Zucman-Rossi J: Transcriptome classification of HCC is related to gene alterations and to new therapeutic targets. Hepatology 45(1): 42-52, 2007.

22 Menon S, Yecies JL, Zhang HH, Howell JJ, Nicholatos J, Harputlugil E, Bronson RT, Kwiatkowski DJ and Manning BD: Chronic activation of MTOR complex 1 is sufficient to cause hepatocellular carcinoma in mice. Sci Signal 5(217): ra24, 2012.

23 Samarin J, Laketa V, Malz M, Roessler S, Stein I, Horwitz E, Singer S, Dimou E, Cigliano A, Bissinger M, Falk CS, Chen X, Dooley S, Pikarsky E, Calvisi DF, Schultz C, Schirmacher P and Breuhahn K: PI3K/AKT/MTOR-dependent stabilization of oncogenic far-upstream element binding proteins in hepatocellular carcinoma cells. Hepatology 63(3): 813-826, 2016.

24 Goetz MP, Toft DO, Ames MM and Erlichman C: The HSP90 chaperone complex as a novel target for cancer therapy. Ann Oncol 14(8): 1169-1176, 2003.

25 Patki JM and Pawar SS: HSP90: Chaperone-me-not. Pathol Oncol Res 19(4): 631-640, 2013.

26 Chiosis G, Vilenchik M, Kim J and Solit D: HSP90: The vulnerable chaperone. Drug Discov Today 9(20): 881-888, 2004.

27 Neckers L: Using natural product inhibitors to validate HSP90 as a molecular target in cancer. Curr Top Med Chem 6(11): 1163-1171, 2006.

28 Zhang $\mathrm{H}$ and Burrows F: Targeting multiple signal transduction pathways through inhibition of HSP90. J Mol Med (Berl) 82(8): 488-499, 2004.
29 Pascale RM, Simile MM, Calvisi DF, Frau M, Muroni MR, Seddaiu MA, Daino L, Muntoni MD, De Miglio MR, Thorgeirsson SS and Feo F: Role of HSP90, CDC37, and CRM1 as modulators of p16(INK4A) activity in rat liver carcinogenesis and human liver cancer. Hepatology 42(6): 1310-1319, 2005.

30 Yoo SH, Kim HY, Rho JH, Jeong SY, Yun J, Yun I, Park HT and Yoo YH: Targeted inhibition of mitochondrial HSP90 induces mitochondrial elongation in Hep3B hepatocellular carcinoma cells undergoing apoptosis by increasing the ROS level. Int $\mathbf{J}$ Oncol 47(5): 1783-1792, 2015.

31 Zhao S, Li H, Jiang C, Ma T, Wu C, Huo Q and Liu H: 17Demethoxy-reblastatin, an HSP90 inhibitor, induces mitochondria-mediated apoptosis through down-regulation of MCL1 in human hepatocellular carcinoma cells. J Bioenerg Biomembr 47(5): 373-381, 2015.

32 Gibson BA and Kraus WL: New insights into the molecular and cellular functions of poly(adp-ribose) and PARPs. Nat Rev Mol Cell Biol 13(7): 411-424, 2012.

33 Rouleau M, Patel A, Hendzel MJ, Kaufmann SH and Poirier GG: PARP inhibition: PARP1 and beyond. Nat Rev Cancer 10(4): 293-301, 2010.

34 Horton JK and Wilson SH: Predicting enhanced cell killing through PARP inhibition. Mol Cancer Res 11(1): 13-18, 2013.

35 Tangutoori S, Baldwin P and Sridhar S: Parp inhibitors: A new era of targeted therapy. Maturitas 81(1): 5-9, 2015.

36 Basu B, Sandhu SK and de Bono JS: PARP inhibitors: Mechanism of action and their potential role in the prevention and treatment of cancer. Drugs 72(12): 1579-1590, 2012.

37 Lai K and Elsas LJ: Overexpression of human UDP-glucose pyrophosphorylase rescues galactose-1-phosphate uridyltransferase-deficient yeast. Biochem Biophys Res Commun 271(2): 392-400, 2000.

38 Kovacs K, Decatur C, Toro M, Pham DG, Liu H, Jing Y, Murray TG, Lampidis TJ and Merchan JR: 2-Deoxy-glucose downregulates endothelial AKT and ERK via interference with $\mathrm{N}$-linked glycosylation, induction of endoplasmic reticulum stress, and GSK3beta activation. Mol Cancer Ther 15(2): 264$275,2016$.

39 Song KH, Kang JH, Woo JK, Nam JS, Min HY, Lee HY, Kim $\mathrm{SY}$ and $\mathrm{Oh} \mathrm{SH}$ : The novel IGF-IR/AKT-dependent anticancer activities of glucosamine. BMC Cancer 14: 31, 2014.

40 American Cancer Society. Available from: http://www. cancer.org/cancer/livercancer/detailedguide/liver-cancer-treatingtargeted-therapy
Received September 26, 2016

Revised October 11, 2016

Accepted October 17, 2016 